\title{
Reservoir Evaluation from an Automatic Saturation Inversion Algorithm Using TLIL Data
}

\author{
Zhenhua Liu \\ Mechanical Engineering College \\ Xi' an Shiyou University \\ Xi' an, 710065, China \\ e-mail: liuzhenhua@xsyu.edu.cn
}

\begin{abstract}
Invasion of drilling mud filtrate into a reservoir is related to time after the bed was reached, so that logging-tool response is a function as time. Traditional algorithms of resistivity inversion are based on a simplified step-invasion model that is independent on time, hence it is difficult to obtain the dynamical image of a realistic invasion process and the accuracy of inversion is affected. An automatic inversion algorithm of time-lapse induction $\log$ (TLIL) is modeled with fluid- and formation-related parameters that control the dynamic invasion process. It is able to characterize the dynamic invasion process realistically and to provide more information for log analysts to evaluate reservoirs. From matching simulation results with time dependent field logging data, the present model can yield more reliable estimates of formation properties such as radial heterogeneities resulting from invasion, original fluid parameters, etc. Field applications for dual-induction measurement illustrate that the method is reasonable and efficient for evaluation of reservoirs.
\end{abstract}

Keywords-Well Logging; Inversion; Time; Resistivity; Saturation

\section{INTRODUCTION}

The aid of electrical-logging data inversion is to determine true-formation resistivity, furthermore to estimate original hydrocarbon saturation. However, environment effects (borehole, shoulders, invasion, tool eccentricity, etc.) influence the measurements. For a thick bed, the effect of drilling mud filtrate invading into a reservoir on logging responses is usually significant for a permeable and porous formation; hence, invasion correction is introduced by charts or computer inversion routines. Although charts and computer auto-inversion techniques have been developed greatly, most of them are based on the step-profile invasion model, which presumes a piston-like displacement of reservoir fluid by filtrate and the resistivity profile changes abruptly at the boundary between invaded zone and uninvaded formation [1]. Invaded zone is marked by an invasion depth $r_{i}$ and a unique resistivity $R_{x o}$ usually. The formation resistivity varies radially from $R_{x o}$ to trueformation resistivity $R_{t}$ in a single step.

In fact, a realistic invasion process is much complicated. First, it is dynamical. Filtrate propagates into the formation gradually with time after the formation is opened. Second, invasion profile does not move as the piston-like version due to the fluid mixing and ion diffusing between filtrate and connate water in formation. Fluid- and formation-related parameters have various values at different positions in radial direction [1,2]. Therefore, the oversimplified step-profile invasion model will lead to the deviation of inverted formation parameters from true formation.

Dynamic variation of invasion profiles affect on $\log$ measurements significantly. It results in that the logging readings at the early invasion period may be very different from the response at a later invasion stage. Therefore, the measurements are time dependent. The dynamic invasion and time-related resistivity $\log$ (i.e. time-lapse resistivity logs) have been modeled using fluid flowing equations and logging theory [3-6]. Since the calculation of time-lapse logs deals with fluid- and formation-related parameters that control the dynamic invasion process, trial-and-error forward modeling had been used to fit the field data historically in order to estimate permeability $[3,4]$, saturation and true-formation resistivity $[5,6]$. However this method is manual and requires modifying the initial guesses repeatedly. Its result depends on the operator's experience strongly as well. Automatic inversion technique of timelapse log data is suggested in the present study, it makes the logging interpretation become much accurately and objectively.

Usually, only two parameters, invasion depth $r_{i}$ and true-formation resistivity $R_{t}$, are inverted from dualinduction or dual-laterolog measurements using traditional inversion algorithm based on the step-profile invasion model because they caused two measurement data. Invadedzone resistivity is determined from another logging tool with a shallow depth of investigation such as latrerolog 8 [7]. Original reservoir image and invasion process are difficulty to be recovered and characterized by traditional inversion routine from the limited log data.

For time-lapse logging, a well is logged twice at least; thus four or more data are recorded from a dual-induction or 
a dual-laterolog tool. Time-lapse logging data contain more information about reservoir and dynamic invasion process. The present paper proposes an auto-inversion model of time-lapse induction log (TLIL) dada. Original reservoir parameters and dynamic invasion image is physically true to be obtained using the present inversion of time-lapse log data. The present inversion yields the reasonable saturation estimation and suggests a realistic dynamic invasion image for reservoir evaluation. Case study in an oilfield of China was also illustrated.

\section{FORWARD MODEL AND TIME-LAPSE LOGGING RESPONSES}

The forward model consists of dynamic invasion equations and resistivity logging theory. The typical behaviors of dynamic invasion process and time-lapse log were also illustrated in this section.

\section{A. Dynamic invasion process}

The first step of time-lapse logging algorithm is based on dynamic invasion model. During drilling, mud filtrate pours into formation under the drive force of pressure differential between mud column and formation. Invasion is also related to formation permeability, porosity, original water saturation, native-water salinity, mud-filtrate salinity, viscosity of fluids, capillary pressure, and so on. At the beginning of bit penetration, mud cake is built up within a short time period, and then the mud-cake permeability controls the invasion [3]. With the extension of invading geometry area and the building of mud cake, invasion rate decreases with time; hence the invasion profile changes at various time stages. The dynamic invasion process can be calculated from fluid flow equations $[3,5]$.

In the present dynamic invasion model, thick beds are studied; thus shoulder effect is negligible. Filtrate invades into a formation radially and displaces the native fluids, which have pressure-dependent compressibility and viscosity. The displacement between filtrate and hydrocarbon is immiscible. Gravity is ignored. More details on dynamic invasion modeling and numerical method had been discussed in author's prior literatures $[5,6]$.

The model produces following dynamic invasion characteristics: (1) formation water saturation $S_{w}(r, t)$ that varies with time after bit penetration and is a function of both time $t$ and radial distance $r$; (2) water salinity profile $\mathrm{C}_{w}(r, t)$ that change due to the mixing of filtrate with native water by the driving force of salinity difference between filtrate and formation water; (3) formation-water resistivity $R_{w}(r, t)$ that is related to salinity and formation temperature; (4) formation resistivity distribution $R_{f}(r, t)$ that satisfies Archie's water-saturation equation:

$$
R_{f}(r, t)=\frac{a R_{w}(r, t)}{S_{w}^{n}(r, t) \varphi^{m}}
$$

Where $\varphi$ is porosity, $m$ the porosity exponent, $n$ the saturation exponent, and $a$ is a constant.
The general behavior of dynamic invasion is illustrated in Fig.1, which shows the radial formation-resistivity profile at 3.7, 7.9, 16.7, and 33.4days after drilling. The vertical axis denotes formation resistivity $R_{f}$ and horizontal axis is invasion time. The main field conditions were: original water saturation $S_{w}=0.5$, formation absolute permeability $k=0.002 \mu \mathrm{m}^{2}$, porosity $\varphi=0.1$, mud salinity $C_{m f}=5000 \mathrm{mg} / \mathrm{L}$, formation-water salinity $C_{w}=130000 \mathrm{mg} / \mathrm{L}$, $m=n=2$ and $a=1$ in (1). The pressure differential between borehole and initial formation $\triangle P=5 \mathrm{MPa}$.

Fig.1 illustrated that there is not a sharp boundary between invaded zone and uninvaded formation. A lowresistivity annulus in the front of invaded zone forms because high-salinity formation water $\left(C_{w}=130000 \mathrm{mg} / \mathrm{L}\right)$ is displaced by low-salinity filtrate $\left(C_{m f}=5000 \mathrm{mg} / \mathrm{L}\right)$. The annulus is not usually be modeled by traditional inversion algorithm based on step invasion version [1]. The resistivity invasion profile moves toward the depth of formation with a reduced rate owing to the extending of invasion geometry.

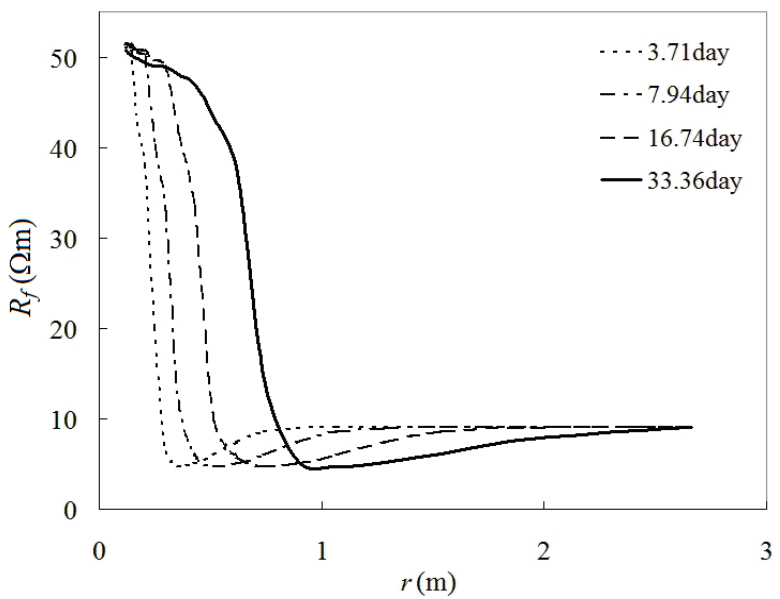

Figure 1. Dynamic invasion profile of formation resistivity at various time stages

\section{B. Time-lapse Induction Log}

The variation of resistivity profiles with time causes a notable impact on apparent resistivity of log tools. It is easy to understand that the resistivity logging measurements depart from the actual formation parameter and they are not constants, but variable data depending on the time when the well was logged. A simple and rapid method to calculate induction-logging response is geometry factor theory, which generated the medium- and deep-induction response, $R_{I M}$ and $R_{I D}$, respectively:

$$
\begin{aligned}
R_{I M}(t) & =\left[\int_{0}^{\infty} \frac{g_{I M}(r)}{R_{f}(r, t)} d r\right]^{-1} \\
R_{I D}(t) & =\left[\int_{0}^{\infty} \frac{g_{I D}(r)}{R_{f}(r, t)} d r\right]^{-1}
\end{aligned}
$$


Where $g(r)$ is radial differential geometrical factor. The apparent resistivity is determined by resistivity profile and geometry factor at the same time. Different time stages correspond to various log readings, i.e., time-lapse induction $\log$ (TLIL).

Fig. 2 represents a typical effect of filtrate invasion on time-lapse dual-induction log from the present forward modeling. Horizontal axis represents the time after drilling and vertical axis gives medium- and deep-apparent resistivities that were calculated using (2) from resistivity profiles in Fig.1. Since a dual-induction tool consists of shallow and a deep focused array simultaneously, the separation between $R_{I M}$ and $R_{I D}$, in the absence of shoulder effects, is indicative of invasion. Due to the radial variation of resistivity profile with time, the dual-resistivity responses logged early will be different from the values recorded at a later time period; hence the apparent resistivity is a function as invasion time. At the beginning of a bed just be reached, $t=0$, invasion dose not occur and the readings of both medium- and deep-array are essentially the same. They equals to the true-formation resistivity $R_{t}$.

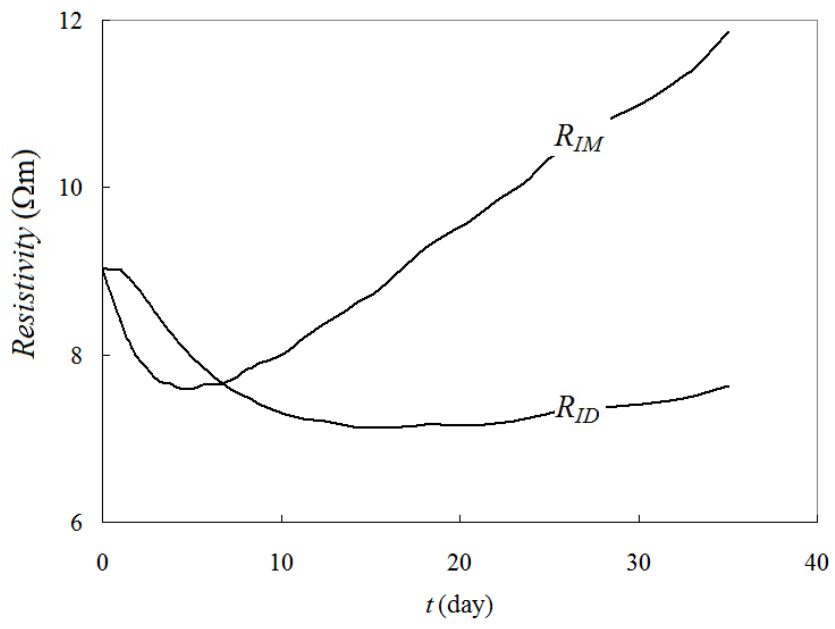

Figure 2. Typical time-lapse dual-induction logging responses

An induction-logging tool is usually used in the case of fresh-water mud filtrate with low salinity invading into a formation, which contains high-salinity formation water. If the volumes in pores in formation are not fully occupied by hydrocarbon, not only the non-conductive oil is displaced by the relatively non-conductive filtrate during invasion, but also the high-salinity, relatively conductive formation water is flushed away. Therefore, a low resistivity annulus forms between the uninvaded formation and the near-wellbore region of the invaded zone (see Figure 1). At early times after bit penetration, the medium-induction response, which has a shallow depth of investigation, is affected by this annulus seriously, as shown in Fig.2. As invasion progresses with time, the annulus propagates into formation and increasingly influences the deep-induction measurement, hence $R_{I D}$ decreases. Meanwhile, since the high-resistivity filtrate fills the volumes where the medium-induction log is focused, $R_{I M}$ increases gradually with time. When the lowresistivity annulus reaches the depth of medium-induction measurement, $R_{I M}$ reaches its minimum value. Likewise, $R_{I D}$ has its minimum value when the annulus migrates to the depth of deep-induction investigation. Responding to the high resistivity region between the low-resistivity annulus and the wellbore, both $R_{I M}$ and $R_{I D}$ increases again after they reached their minimum values respectively. The alternation of time-lapse apparent resistivity exhibits the effect of a dynamic invasion process on electrical-logging measurements.

\section{The cross time}

The cross point in time ax is of curve $R_{I D} \sim t$ with $R_{I M} \sim t$ is known as cross time. It is related to the original water saturation in formation. If a reservoir contains oil and water equivalently, the cross point usually locates at the middle time stage. The result of $R_{I D}>R_{I M}$ will be recorded from logging at the early invasion time. The inverse result, $R_{I M}>R_{I D}$, will be obtained from later measurement.

If a reservoir is hydrocarbon-bearing, little moveable water exists due to the lower original water saturation. High-resistivity oil is displaced by the low-resistivity filtrate, hence the resistivity near the wellbore is less than that of the formation. Because the investigation depth of deepinduction logs is deeper than that of medium-induction logs, the displacement process shows a more sensitive effect on medium-induction measurement than on deep-induction responses. Within a long period of invasion process, $R_{I D}$ is greater than $R_{I M}$. The cross time is late. If a well is not logged at later period, $R_{I D}>R_{I M}$ is often recorded.

If the bed is water-bearing, the low-resistivity native water is displaced by high-resistivity fresh filtrate. The invasion creates a high-resistivity region adjacent to the wellbore. Therefore, the measurement of $R_{I M}>R_{I D}$ is reasonable. With the movement of the invasion front towards the formation, formation water is displaced by filtrate. The result, $R_{I M}>R_{I D}$, occurs continuously. The cross point of medium- and deep-induction measurement is located at $t=0$ (for a water zone) or early invasion period.

Therefore the cross time decreases with the increasing of water saturation. For an oil-bearing reservoir, the cross time is late; and for a water zone, the cross time is located at the early time stage. If the time of a log run is less than the cross time, $R_{I D}>R_{I M}$ will be recorded. If a well is logged later and the logging time addresses beyond the cross time, $R_{I D}>R_{I M}$ will occur. The characteristics can be used to evaluate a reservoir qualitatively [8]. However, for an automatic and quantitative analysis, a computer inversion algorithm is proposed in the following section.

\section{INVERSION ALGORITHM}

The target of resistivity logging interpretation is estimation of original water or oil/gas saturation from the determination of true-formation resistivity through field log data. Therefore the original water saturation $S_{w}$ is an 
unknown parameter in the present inversion technique of time-lapse dual-induction log. On the other hand, the timelapse resistivity response is governed by the variation of resistivity profile. Radial heterogeneity of formation and the difference between $R_{I M}$ and $R_{I D}$ are related to invasion rate that is controlled by mud-cake permeability $k_{m c}$ [9], which is difficulty to be determined, so that it is set to be an unknown parameter as well in the present invasion process. Thus, two unknown parameters, $S_{w}$ and $k_{m c}$, are inverted in the present inversion model.

As an adaptive algorithm, least-squares theory is widespreadly applied in the inversion of resistivity logs $[10,11]$. If an estimated initial water saturation $S_{w}^{0}$, an estimated mud-cake permeability $k_{m c}^{0}$, and vector $\boldsymbol{G}^{\mathbf{0}}$ of initial modeling parameters cause the model response $R_{i j}^{0}=R_{i j}\left(S_{w}^{0}, k_{m c}^{0}, \boldsymbol{G}^{0}\right)$ for medium-induction $\log (j=1)$ and deep-induction $\log (j=2)$ at $i$-th $\log$ performance, the model responses $R_{i j}$ for the data $S_{w}, k_{m c}$ and $\boldsymbol{G}$ is

$$
\begin{aligned}
& R_{i j}\left(S_{w}, k_{m c}, \boldsymbol{G}\right)=R_{i j}^{0}\left(S_{w}^{0}, k_{m c}^{0}, \boldsymbol{G}^{0}\right)+ \\
& +\left.\frac{\partial \boldsymbol{R}_{i j}\left(S_{w}, k_{m c}, \boldsymbol{G}\right)}{\partial S_{w}}\right|_{S_{w}=S_{w}^{0}, k_{m c}=k_{m c}^{0}} ^{\boldsymbol{G}=\boldsymbol{G}^{\mathbf{0}}} \delta S_{w}+ \\
& +\left.\frac{\partial \boldsymbol{R}_{i j}\left(S_{w}, k_{m c}, \boldsymbol{G}\right)}{\partial k_{m c}}\right|_{S_{w}=S_{w}^{0}, k_{m c}=k_{m c}^{0}} ^{\boldsymbol{G}=\boldsymbol{G}^{\mathbf{0}}} \delta k_{m c}
\end{aligned}
$$

Field log data were written as a column vector:

$$
\mathbf{R}=\left(R_{11}, R_{12}, R_{21}, R_{22}, \ldots\right)^{\mathrm{T}}
$$

The calculated apparent resistivity as a column vector:

$$
\mathbf{R}^{\prime}=\left(R_{11}^{\prime}, R_{12}^{\prime}, R^{\prime}{ }_{21}, R_{22}^{\prime}, \ldots\right)^{\mathrm{T}}
$$

From (3), a matrix equation

$$
\Delta \mathbf{R}=\mathbf{R}-\mathbf{R}^{\prime}=[\mathbf{J}] \Delta \mathbf{P}
$$

was obtained, where vector $\Delta \mathbf{R}$ is the difference between measured data and apparent resistivity calculated from the initial guess, $\Delta \mathbf{P}$ is the increment of unknown parameter column vector $\mathbf{P}=\left(S_{w}, k_{m c}\right)^{\mathrm{T}}$. [J] is Jacobi matrix.

For $i$-th logging measurement, the element in the 1 st and 2nd column of Jacobi matrix are

$$
J_{i 1}=\frac{\partial R_{i j}}{\partial S_{w}}, \quad J_{i 2}=\frac{\partial R_{i j}}{\partial k_{m c}}
$$

where $j=1$ and 2 denote medium- and deep-induction measurement respectively.
To prevent the instability of increment $\Delta \mathbf{P}$, the step size is adjusted, in which a truncation parameter $\alpha$ is introduced during the calculation

$$
\Delta \mathbf{P}=\left([\mathbf{J}]^{+}[\mathbf{J}]+\alpha[\mathbf{I}]\right)^{-1}[\mathbf{J}]^{\mathbf{T}} \Delta \mathbf{R}
$$

where $[\mathbf{I}]$ is identity matrix. From (4)-(6), increment $\Delta \mathbf{P}$ is solved and the new unknown parameter column vector $\mathbf{P}^{\prime}$ is obtained

$$
\mathbf{P}^{\prime}=\mathbf{P}_{\mathbf{0}}+\Delta \mathbf{P}
$$

Then the new data $\mathbf{P}^{\prime}$ is inputted to the algorithm to generate new modeling responses and new increment $\Delta \mathbf{P}$. The iteration of (4)-(7) is cycled until the calculated instrument responses agree with field data.

Time-lapse dual-induction logs provide four field data for twice measurements or six readings if a well is logged three times. They are enough to invert the two unknown parameters: $S_{w}$ and $k_{m c}$.

\section{FIELD APPLICATION}

The present case study came from an oil field in China. The well was logged three times at 4, 10 and 20day respectively after the formation was penetrated by the drill bit. Resistivity measurements from all three logging passes exhibited varying responses to invasion (see Table 1). The first and second measurements suggested $R_{I D}>R_{I M}$; then the third logging showed $R_{I D}<R_{I M}$. Therefore the cross time is located between 10day and 20day. According to the analysis in Section IIC, it is concluded that this reservoir would contain oil and water, perhaps mainly oil because the cross time is late.

TABLE 1 A field data inversion example

\begin{tabular}{|c|c|c|c|}
\hline & $\begin{array}{c}\text { Field data } \\
(\mathbf{\Omega} \mathbf{m})\end{array}$ & $\begin{array}{c}\text { Inversion } \\
(\mathbf{\Omega} \mathbf{m})\end{array}$ & $\begin{array}{c}\text { Relative } \\
\text { error }\end{array}$ \\
\hline 1st logging & $R_{I M}=3.0$ & $R_{I M}=3.054$ & $1.8 \%$ \\
(at 4day) & $R_{I D}=3.5$ & $R_{I D}=3.550$ & $1.4 \%$ \\
\hline 2nd logging & $R_{I M}=2.8$ & $R_{I M}=2.767$ & $-1.2 \%$ \\
(at 10day) & $R_{I D}=3.0$ & $R_{I D}=3.114$ & $3.8 \%$ \\
\hline 3st logging & $R_{I M}=2.9$ & $R_{I M}=2.878$ & $-0.7 \%$ \\
(at 20day) & $R_{I D}=2.7$ & $R_{I D}=2.735$ & $1.3 \%$ \\
\hline Initial guesses & \multicolumn{3}{|c|}{$S_{w}^{0}=0.33, k_{m c}^{0}=6.3 \times 10^{-6} \mu \mathrm{m}^{2}$} \\
\hline Inversion results & \multicolumn{3}{|c|}{$S_{w}=0.2, k_{m c}=1.00 \times 10^{-6} \mathrm{~m}^{2}$} \\
\hline
\end{tabular}

True-formation resistivity and original water saturation were inverted from the present inversion algorithm. Core analysis and well testing suggested that: $k=0.32 \mu \mathrm{m}^{2}, \varphi=0.21$, $C_{m f}=5000 \mathrm{mg} / \mathrm{L}, C_{w}=120000 \mathrm{mg} / \mathrm{L}, m=1.84, n=1.56, a=0.93$, $T=60^{\circ} \mathrm{C}$, and $\triangle P=4.0 \mathrm{MPa}$. Results from the present inversion were drawn in Fig.3 and Fig.4. Fig.3 illustrated three formation resistivity profiles that corresponded to the three measurements at 4, 10 and 20day respectively. Figure 4 was the time-lapse dual-induction apparent resistivity as functions of time along with the field data.

The present inverted original water saturation, 0.33 , suggests that the reservoir pores is dominated by oil. It is 
very close to the results of quantitative logging interpretation and well testing analysis. The true-formation resistivity $R_{t}$ determined by (1), $3.6 \Omega \mathrm{m}$, is much higher than field $\log$ data. The log readings were influenced by invasion strongly due to the low-resistivity mud filtrate, thus lowresistivity invasion zone. Deep-induction measurement $R_{I D}$ was only $97 \%$ of $R_{t}$ after the reservoir was immersed for 4 day, then $83 \%$ at 10 day, and finally $75 \%$ at 20 day. The medium induction reading decline more, as shown in Fig.4. Obvious deviation would be introduced if the reservoir was immersed for a long period.

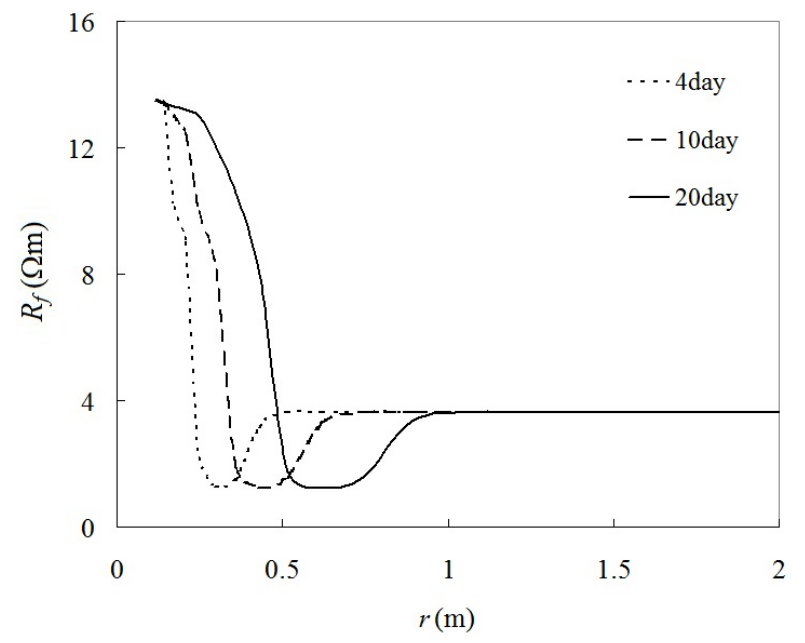

Figure 3. Inversion resistivity profiles

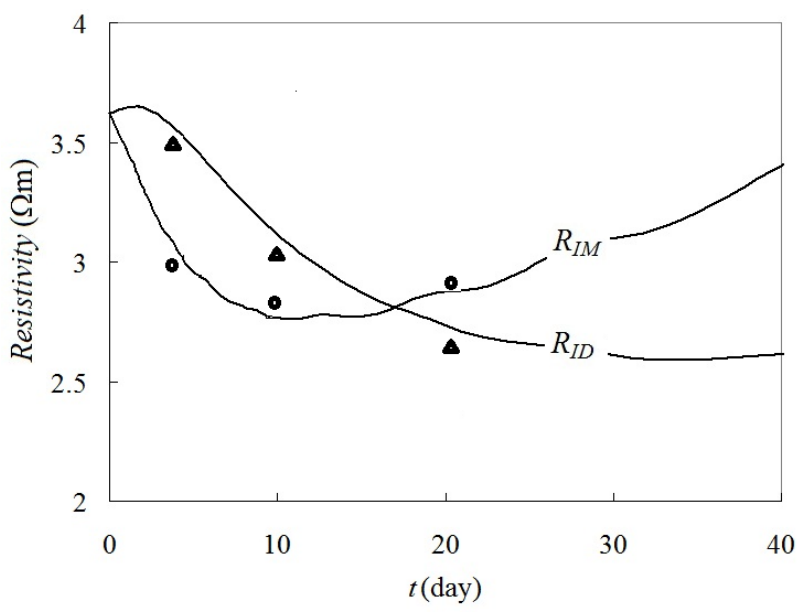

Figure 4. Inversion TLIL responses (solid lines) and field log data (triangles for and $R_{I D}$ and circles for $R_{I M}$ )

\section{DISCUSSIONS}

Common combining resistivity logging tools usually provide three curves with shallow-, medium-, and deepinvestigation depths, hence 3-parameter $\left(R_{x o}, r_{i}\right.$ and $\left.R_{t}\right)$ inversion are realized using invasion correction charts or traditional inversion algorithm based on step-profile invasion model, yet the low-resistivity annulus in the front of invasion zone is neglected. Difference between the ideal invasion model and a realistic invasion process will introduce the deviation of estimated formation resistivity from true value. Especially for the high contrast of formation-water salinity and filtrate salinity, the effect of low-resistivity annulus on measurement becomes so significant that error might be introduced if the piston-like invasion version is modeled.

The physical understanding of invasion process is importance of improving the inversion results. Repeating resistivity measurements at different time stages are helpful to better understand and quantify the invasion process. From the discussion about Figure 2, the low resistivity annulus in the front of invaded zone plays an important role to log readings, but it cannot be inverted from dual-induction or dual-laterolog responses based on step-invasion model. If we intend to characterize the invaded zone and lowresistivity annulus simultaneously using the ideal stepprofile invasion style, it needs at least five parameters: radius of invaded zone, resistivity in invaded zone, width of the low-resistivity annulus, resistivity in the annulus, and true-formation resistivity. However a dual-induction or a dual-laterolog tool provides only two readings from a logging run, it is not enough to determine the five unknown parameters. Although a new array induction tool (AIT) or [12] multi-laterolog device that provide five data with different investigation depths makes the inversion of the low-resistivity annulus become possible, it still cannot suggest the dynamic evolution of invasion process. Using time-lapse logging, we can recovery the original formation data and exhibit the dynamic evolution of a realistic invasion process. Therefore, if the resistivity inversion is conditioned by both the dynamic invasion process and the field log data recorded at different time stages, it is possible to invert the original formation parameters with less bias and to suggest the dynamic evolution of invasion profiles. Of cause, if time-lapse AIT log or multi-laterolog device are performed, the inversion results based on the present principle will be much accurate.

Since time-lapse $\log$ is related to formation data, fluid characters, mud property, pressure differential between borehole and original bed, and so on, it can be used to describe the realistic invasion process. However, if these supporting input data are not sufficient or not available, the accuracy of calculation will be influenced. In addition, if a well was logged only once, it is difficult to determine the variation of apparent resistivity with time accurately; hence non-unique solutions of formation model might be generated. It is therefore suggested that a well would be logged at least twice to avoid the non-unique solutions in the present inversion of time-lapse log. Logging three or more times is better, as shown in Figure 4, but is of cause more expensive. Usually, logging twice is enough for the utilization of present model, because each measurement yields two resistivity readings with different depths of investigation using a dual-induction instrument; thus, four readings are recorded from twice log. They are sufficiently to invert two unknown parameters, original water saturation and mud-cake permeability. If the each group of measured data is inputted to a traditional inversion routine based on step-profile invasion model respectively, different inverted 
results might be resulted from the various objective functions. Compared with conventional inversion technique, the time-lapse logging inversion holds all field log data recorded at varying time in its algorithm. Moreover, one of an important character of the present model is that other parameters, such as permeability, porosity, etc, could also be inverted according to the present principle.

In this algorithm, the mud-cake permeability is taken to be a constant. In fact, $k_{m c}$ decreases with time $t$ at the beginning of bit penetration; then it tends to a constant during the later invasion time stage [3]. However, the relationship between $k_{m c}$ and $t$ is hard to be determined. The present constant $k_{m c}$ can be regarded as the average contribution of mud-cake permeability to the invasion process. If the variation of $k_{m c}$ with $t$ is known for a well, it can be programmed and used to invert original water saturation and other unknown parameters such as permeability, porosity, etc.

\section{CONCLUSIONS}

Dynamic invasion process causes that the resistivity responses of a well logging tool are time dependent. And the log data deviate from true-formation resistivity. The immersed time and dynamic invasion characteristics ought to be included in inversion problems.

The present inversion model of time-lapse logging is suitable to suggest dynamic invasion profiles, to determine original water saturation and true-formation resistivity.

Compared with traditional 2-parameters $\left(r_{i}\right.$ and $\left.R_{t}\right)$ inversion methods, the present inversion technique utilizes all log data recorded at various invasion stages; hence, it is capable of inverting realistic invasion profiles and formation data efficiently with less bias.

The physical principle can be extended to other resistivity logging methods [13], such as a dual-laterolog tool, an array induction tool, and even an array laterolog instrument. It provides a new viewpoint to invasion effect and resistivity inversion method.

\section{ACKNOWLEDGMENT}

The authors would like to thank Prof. J. Ouyang for his help with the field data and Prof. J. H. Zhang for the helpful discussions.

\section{REFERENCES}

[1] G. Ankur, T. Carlos, V. Ben, G. F. Seminario. G., Johnny, "Correction of invasion effects on well logs in Camisea gas reservoirs, Peru, with the construction of static and dynamic multilayer petrophysical models", AAPG Bulletin, vol. 97(3), pp.379-412, 2013

[2] K. Ling, H. Zhang, Z. Shen, A. Ghalambor, G. Han, J. He, P, Peng, "A new approach to estimate invasion radius of water-based-drillingfluid filtrate to evaluate formation damage caused by overbalanced drilling", SPE Drill. Complet, vol.30(1), pp. 27-37, 2015

[3] D. P. Tobola and S. A. Holditch, "Determination of reservoir permeability from repeated induction logging", SPE Formation Evaluation, pp20-27, March, 1991

[4] C. Y. Yao and S. A. Holditch, "Reservoir permeability estimation from time-lapse log data", SPE Formation Evaluation, pp.69-74, June, 1996.

[5] J. H. Zhang, Q. Hu and Z. H. Liu, "Estimation of true formation resistivity and water saturation with a time-lapse induction logging method", The Log Analyst, Vol.40(2), pp38-148, 1999

[6] Z. H. Liu, J. Oyang, and J. H. Zhang, "Dynamic Dual-Laterolog Responses: Model and Field Applications in the Bohai Gulf of China", Journal of Petroleum Science and Engineering, vol.23(1-2), pp.1-11, 1999.

[7] S. X. Du, C. W. Yang and L. B. Hu, "Evaluating Reservoir Quickly With Dual Induction-8 Laterolog Data", Well Logging Technology (in Chenese), vol.28(4), 327-328, 2004

[8] J. M. Sun, S. Y. Zheng, "A new method for connate water saturation calculation using time-lapse logging data", Journal of Petroleum Science and Engineering, vol.50(3-4), pp. 204-210, 2006.

[9] A. E. Osgouei, M. H. Ozyurtkan and G. Altun, "Dynamic filtration properties of fresh water sepiolite-based muds", Energy Sources, Part A, vol.36(19), pp. 2079-86, 2014

[10] M. Arioli and S. Gratton, "Linear regression models, least-squares problem, normal equations, and stopping criteria for the conjugate gradient method", Computer Physics Communications, vol.183(11), pp.2322-2336, 2012

[11] J. Crassidis, Y. Cheng, "Error-covariance analysis of the total leastsquares problem", Journal of Guidance, Control, and Dynamics, vol.37(4), pp. 1053-1063, 2014

[12] C. Bill, K. Rashid, M. H. Martin, J. Z. Li, D. Xiao, S. K. Yang, “A study of differences in array induction and multi-laterolog responses in a well drilled with high-salinity water-based mud",

[13] F. Bellmunt, A. Marcuello, J. Ledo, P. Queralt, E. Falgqas, B Benjumea, V. Velasco, E. Vazqez-Sune, "Time-lapse cross-hole electrical resistivity tomography monitoring effects of an urban tunnel", Journal of Applied Geophysics, vol.87, pp.60-70, 2012. 